Bull. Mater. Sci., Vol. 2, Number 3, August 1980, pp. 161-165. (C) Printed in India.

\title{
Preparation of rare earth-cobalt magnet alloy by reduction-diffusion process
}

\author{
T S KRISHNAN \\ Metallurgy Division, Bhabha Atomic Research Centre, Trombay, Bombay 400085 , \\ India
}

MS received 29 April 1980

\begin{abstract}
Preparation of rare earth-cobalt alloys by reduction-diffusion (R-D) process is described. The process essentially involves mixing of the rare earth oxide and cobalt/cobalt oxide powders in proper proportion and high temperature reduction of the charge in hydrogen atmosphere, followed by aqueous leaching of the reduced mass to yield the alloy powder. Comparison is made of the magnetic properties of the R-D powder with those of the powder prepared by the direct melting (DM) route and it is observed from the reported values for $\mathrm{SmCo}_{5}$ that the energy product of the R-D powder ( $\sim 22 \mathrm{MGOe})$ is only marginally lower than that of the Directly Melted alloy ( $\sim 25 \mathrm{MGOe})$. The paper also includes the results of studies carried out at the Bhabha Atomic Research Centre on the preparation of misch metal-cobalt alloy by the R-D process.
\end{abstract}

Keywords. Rare earth alloys; permanent magnets; rare earth-cobalt magnets; reduction-diffusion.

\section{Introduction}

Interest in the rare earth-cobalt systems stemmed from the studies of Strnat and Hoffer, who in the year 1966 discovered attractive permanent magnet properties in Y-Co alloys (Strnat and Hoffer 1966). In the following year Strnat and coworkers (Strnat et al 1967) made an extensive study of the binary systems of cobalt and several rare earth metals, which unfolded a family of new permanent magnet materials. Some of these alloys, e.g., Sm-Co and $\mathrm{Pr}-\mathrm{Co}$, having 1 : 5 stoichiometry have later on been shown to possess maximum energy products, almost three times that of the best conventional permanent magnets made of platinumcobalt alloys. The existence of large raw material resources for the rare earths in the world and the development and application of modern techniques for separation of individual rare earth elements gave further impetus for intense research in the rare earth $-3 d$ transition metals systems.

There are four major possible routes for the production of rare earth-cobalt alloys. These are (1) arc melting, (2) induction melting, (3) electro-winning, and (4) reduction-diffusion (R-D). Of these, the first two methods are well established and widely practised. These processes are simple to operate and yield alloys of the highest purity. However, both these processes require the 
alloy constituents in their elemental form, and hence are very expensive. Besides, handling of the highly reactive rare earth metals also calls for special handling facilities, adding to the capital and operational costs. The electro-winning and the reduction-diffusion processes, on the other hand, are both based on much cheaper raw materials and are also less capital intensive. The former, besides, offers the advantage of semi-continuous operation, but yields alloys of only hypostoichiometric (rare earth rich) composition and hence, is not widely practised. The latter, which was developed by Cech (1973) has now found wide acceptance as a production route for rare earth magnet alloys. The process is less capital intensive and easy to operate and straightaway yields alloys of the desired composition. The paper discusses the details of the $R-D$ process and presents the results of some of the earlier works and of the studies carried out at the Bhabha Atomic Research Centre, India.

\section{Experimental}

The Reduction Diffusion process, as the name indicates, involves two stepsreduction and diffusion. In the reduction step, the rare earth compound, say oxide, is reduced to metal with a suitable reductant. The reduced metal then forms alloy with the cobalt metal in the charge by mutual diffusion. The heat balance of the reaction is such that the alloy is obtained directly in the powder form, eliminating the need for the costly milling step required in the other process flow-sheets.

Calcium and thorium are the only metals capable of reducing the rare earth oxides. Of the two, the choice naturally falls on calcium, which is cheaper and does not pose any health problems. Besides, the removal of the calcia slag is fairly easy and can be accomplished by simple aqueous leaching.

The R-D process essentially involves thorough mixing of the reactants-rare earth oxide, cobalt and calcium/calcium hydride-and high temperature soaking of the charge, followed by aqueous leaching of the reduced mass to remove excess calcium and calcium oxide. The amounts of rare earth oxide and cobalt are adjusted to correspond to an alloy composition of $1: 5$ stoichiometry.

A variation of the $R-D$ process was examined by Domazer in which part of the cobalt was replaced by cobalt oxide $\left(\mathrm{CO}_{3} \mathrm{O}_{4}\right)$ (Domazer 1973). As the process involves simultaneous reduction of rare earth and cobalt oxides, Domazer called this the 'Co-reduction process'.

The reduction studies carried out at the Bhabha Atomic Research Centre were confined to mixed rare earth oxides. The reductant calcium was used in the form of granules $(+10 \mathrm{mesh})$ while the particle size of cobalt powder was varied to study its influence on the particle size distribution in the final alloy powder. The reduction temperature and time and the calcium percentage in the charge were also varied to arrive at the optimum conditions.

Weighed amounts of rare earth oxide, calcium and cobalt were blended in a bottle by tumbling and the charge transferred to an alumina boat. The boat was then placed in an alumina tube, which was evacuated and back filled with hydrogen gas. Heating was done in a platinum resistance furnace and a nominal flow of hydrogen was maintained throughout the course of reduction. The reduced 
mass, when cooled to room temperature was taken out and exposed to moist nitrogen for controlled oxidation of the unreacted calcium and slaking of the calcium oxide formed. The resultant powder was then repeatedly washed with de-ionised water and finally with $10 \mathrm{v} / 0$ acetic acid to remove the last traces of calcium. The alloy powder was then washed free of acid and rinsed with acetone prior to vacuum drying. The powder was then classified and evaluated for its magnetic properties.

\section{Results and discussion}

Encouraging results have been reported by Martin and others from their studies on R-D Sm-Co alloys (Martin et al 1973). Some of their important findings are presented in table 1. It will be seen from the table that the magnetic properties of the R-D powder are only marginally lower than those of the powder prepared by the direct melting route. Another interesting observation is that when samarium is partially substituted by praseodymium the properties are even better than those of pure Sm-Co magnets. In all these experiments, the authors have employed liquid phase sintering prior to evaluation of magnetic properties, using R-D/DM powder of $\mathrm{RCo}_{5}$ composition as base (80-90\%) and a rare earth-rich Co-alloy as additive, prepared by the direct melting route.

Table 1. Magnetic properties of R-D and DM powder magnets (Martin et al).

\begin{tabular}{llccccc}
\hline Process & Composition & $\begin{array}{c}\mathrm{Bs} \\
\mathrm{kG}\end{array}$ & $\begin{array}{l}\mathrm{Br} \\
\mathrm{kG}\end{array}$ & $\begin{array}{l}\mathrm{Hc} \\
\mathrm{kOe}\end{array}$ & $\begin{array}{l}\mathrm{Hci} \\
\mathrm{kOe}\end{array}$ & $\begin{array}{l}(\mathrm{BH})_{\max } \\
\mathrm{MGOe}\end{array}$ \\
\hline & & 10.3 & 10.0 & 9.9 & 38 & 24.6 \\
D.M. & $\mathrm{SmCo}_{5}$ & 9.9 & 9.3 & 9.0 & 24 & 21.1 \\
R.D. & $\mathrm{SmCo}_{5}$ & 10.9 & 10.3 & 10.1 & 17 & 26.0 \\
R.D. & $\mathrm{Sm}_{0.42} \mathrm{Pr}_{0.58} \mathrm{Co}_{5}$ & & & & & \\
\hline
\end{tabular}

Table 2. Impurity content and yield of $\mathrm{SmCo}_{5}$ powders prepared by $\mathrm{R}-\mathrm{D}$ and co-reduction processes (Goldschmidt Nr 35 1975).

\begin{tabular}{llcll}
\hline Process & Yield & $\begin{array}{c}\text { Average particle } \\
\text { size }\end{array}$ & $\%$ oxygen & $\begin{array}{c}\text { Impurities } \\
\% \mathrm{Ca}\end{array}$ \\
\hline R-D & $96 \%$ & 5 micrometer & $2 \cdot 2 \%$ & $1 \cdot 88 \%$ \\
Co-reduction & $98 \%$ & 18.6 micrometer & $0 \cdot 23 \%$ & $0 \cdot 20 \%$ \\
D-M & $90-95 \%$ & Milled to size & $100 \mathrm{PPM}$ & $0 \cdot 05 \%$ \\
\hline
\end{tabular}


In both R-D and co-reduction processes, alloy yields of over $96 \%$ have been reported respectively by Cech (1973) and Domazer (1973) (table 2). As is to be expected, the calcium and oxygen contents of the powders are higher, which explains the somewhat lower values of magnetic properties of these powders.

Similar observations have been made during the studies on the preparation of $\mathrm{MMCo}_{5}$ alloy by R-D process, carried out at the Bhabha Atomic Research Centre. Table 3 shows the influence of soaking temperature and time and of calcium

Table 3. Influence of soaking temperature, and time and of calcium excess on the yield and particle size of $\mathrm{MMCo}_{5}$ alloy powder.

Cobalt powder : -400 mesh $(\sim 10$ microns)

\begin{tabular}{|c|c|c|c|c|c|}
\hline & & \multirow{2}{*}{ Alloy yield } & \multicolumn{3}{|c|}{ Particle size (micrometer) } \\
\hline & & & $>61$ & $61-37$ & $<37$ \\
\hline & $1000^{\circ} \mathrm{C}$ & $\begin{array}{l}\text { Reduction } \\
\text { incomplete }\end{array}$ & & & \\
\hline $\begin{array}{l}\text { Soaking temperature } \\
\text { (2 hours calrium } \\
\text { excess } 40 \%)\end{array}$ & $\begin{array}{l}1100^{\circ} \mathrm{C} \\
1200^{\circ} \mathrm{C} \\
1300^{\circ} \mathrm{C}\end{array}$ & $\begin{array}{l}92 \cdot 8 \% \\
93.5 \% \\
93 \cdot 0 \%\end{array}$ & $\begin{array}{l}20 \% \\
25 \% \\
35 \%\end{array}$ & $\begin{array}{l}30 \% \\
45 \% \\
50 \%\end{array}$ & $\begin{array}{l}50 \% \\
30 \% \\
15 \%\end{array}$ \\
\hline $\begin{array}{l}\text { Soaking time } \\
\left(1100^{\circ} \mathrm{C}\right) \\
\text { Calcium excess } 40 \%\end{array}$ & $\begin{array}{l}1 \mathrm{hr} \\
2 \mathrm{hrs} \\
3 \mathrm{hrs} \\
4 \mathrm{hrs}\end{array}$ & $\begin{array}{l}90 \cdot 0 \% \mathrm{~d} \\
92 \cdot 8 \% \mathrm{~d} \\
93 \cdot 5 \% \\
93 \cdot 2 \%\end{array}$ & $\begin{array}{l}15 \% \\
20 \% \\
30 \% \\
35 \%\end{array}$ & $\begin{array}{l}30 \% \\
30 \% \\
40 \% \\
50 \%\end{array}$ & $\begin{array}{l}55 \% \text { A } \\
50 \% \\
30 \% \\
15 \%\end{array}$ \\
\hline Calcium excess & Nil & $\begin{array}{l}\text { Reduction } \\
\text { incomplete }\end{array}$ & & & \\
\hline$\left(1100^{\circ} \mathrm{C} 2 \mathrm{hrs}\right)$ & $\begin{array}{l}25 \% \\
40 \% \\
50 \%\end{array}$ & $\begin{array}{l}91.5 \% \mathrm{~A} \\
92.8 \% \mathrm{~A} \\
90.0 \%\end{array}$ & $\begin{array}{l}22 \% \\
20 \% \\
22 \%\end{array}$ & $\begin{array}{l}35 \% \\
30 \% \\
30 \%\end{array}$ & $\begin{array}{l}43 \% \\
50 \% \\
48 \%\end{array}$ \\
\hline
\end{tabular}

Table 4. Effect of variation of cobalt particle size and of compaction of charge on the yield and particle size of $\mathrm{MMCo}_{5}$ alloy powder.

\begin{tabular}{lccccc}
\hline & Yield & & \multicolumn{3}{c}{ Particle size \% (micrometer) } \\
\cline { 4 - 6 } & & & $>61$ & $61-37$ & $<37$ \\
\hline $\begin{array}{l}\text { Particle size of } \\
\text { cobalt }\end{array}$ & $\begin{array}{c}-100+150 \\
\text { mesh } \\
-150+200 \\
\text { mesh } \\
-400 \text { mesh }\end{array}$ & $92 \cdot 8 \%$ & 60 & 20 & 20 \\
Without compaction & $93 \cdot 0 \%$ & 30 & 40 & 30 \\
Compaction 30 tsi & $\begin{array}{c}93 \cdot 0 \% \\
\text { Reduction } \\
\text { incomplete } \\
93 \cdot 0 \%\end{array}$ & 20 & 30 & 50 \\
\hline
\end{tabular}


excess in the charge on the yield and particle size distribution of the alloy powder. A reduction temperature of $1100-1200^{\circ} \mathrm{C}$, a soaking period of 2-3 hours and a calcium excess (over stoichiometric) of $40 \%$ may be considered satisfactory from the point of view of yield and particle size distribution.

Effect of variation of cobalt powder particle size and the results of reduction of charge with and without compaction are shown in table 4. It will be observed from the table that although the overall yield remains almost the same despite variation in the particle size of cobalt, the particle size distribution in the alloy powder is highly dependent on the cobalt particle size. Also, compaction of the charge is necessary for completion of reduction and higher alloy yields. This is due to the inability of molten calcium to spread through the charge and lack of close contact between the reduced metal and cobalt powder particles in the uncompacted charge.

The alloy powder was $\mathrm{x}$-ray analysed and found to be rich in $\mathrm{RCo}_{5}$ phase with only negligible amounts of free cobalt. The alloy powder fraction in the size range 8-10 micrometer was field pressed and the magnet so obtained gave a maximum energy product of 2-3 MGOe as against an expected value of 5-6 MGOe. This could be attributed to the instrument limitation which could generate a field of only $4-5 \mathrm{kOe}$ in the gap provided. With higher fields, the powder may be expected to give better results.

\section{Conclusions}

The reduction-diffusion/co-reduction process can be a commercially more viable alternative to the direct melting processes for the production of rare earth-cobalt permanent magnet alloys. The process does not call for heavy equipment investment, is easy to operate and is capable of high yields and precise composition. The only drawback of the process is that the alloy powder has high oxygen and calcium contents. Nevertheless, magnets made from R-D powders are quite comparable to those from DM powders and can be used for most of the applications.

\section{References}

Cech R E 1973 USP 3, 748, 193

Domazer H G 1973 DT-P2, 303, 697

Martin D L, Laforce R P, Rockwood A C, Mc Farland C M and Valentine L 1973 Rep. No. 73 CRD068

Strnat K J and Hoffer G I 1966 ATML-TR-65-446 Airforce Materials Laboratory, Ohio

Strnat K J, Hoffer G, Olson J, Ostertag W and Becker J J 1967 J. Appl. Phys. 381001 